\title{
Uso de Tecnología como Recurso Preponderante en el Aprendizaje a Distancia en tiempos de Confinamiento Social
}

\author{
Use of Technology as a Preponderant Resource in Distance Learning in Times of Social \\ Lock-Down
}

Kristian Armando Pineda-Castillo

\section{(0) EDICIÓN: \#-CIVTAC}

Recibido: 29/diciembre/2020

Aceptado: 29/marzo/2021

Publicado: 16/abril/2021

País

${ }^{1}$ México

\section{IIIII Institución}

${ }^{1}$ Colegio de Bachilleres del Estado de Sinaloa

\section{\Correo Eletrónico \\ 'kristiancobaes@hotmail.com}

(iD) ORCID

'https://orcid.org/0000-0002-4686-3587

\section{Citar así: C APA / IEEE}

Pineda-Castillo, K. (2021). Uso de Tecnología como Recurso Preponderante en el Aprendizaje a Distancia en tiempos de Confinamiento Social. Revista Tecnológica-Educativa Docentes 2.0, 11(1), 89-98. https://doi.org/10.37843/rted.v11i1.197

K. Pineda-Castillo, "Uso de Tecnología como Recurso Preponderante en el Aprendizaje a Distancia en tiempos de Confinamiento Social", RTED, vol. 11, n. ${ }^{\circ}$ 1, pp. 89-98, abr. 2021.

\section{Resumen}

Hoy en día, se trabaja continuamente con un modelo educativo de tipo sistémico-holístico, el cual se encuentra evolucionando constantemente. De este modo, el presente estudio tuvo como objetivo comprender la forma en que las Tecnologías del Aprendizaje y el Conocimiento (TAC) aportaron en la preparación de los jóvenes de bachillerato en el contexto de una educación a distancia a lo largo del periodo de confinamiento del COVID-19. Se apeló al enfoque cualitativo, siguiendo el diseño de Teoría Fundamentada constructivista. Los resultados destacaron que las TAC favorecieron la experiencia de los alumnos de distintas maneras; primeramente, porque las estrategias pedagógicas tuvieron un mayor impacto en el proceso de enseñanza, puesto que se promovieron diversas situaciones encaminando a los bachilleres a desempeñar un papel activo; en segundo lugar, los recursos tecnológicos permitieron administrar con facilidad el aprendizaje por medio de diferentes dispositivos electrónico-digitales; finalmente, el uso de las tecnologías promovió el desarrollo de competencias digitales mediante una interacción constante con aplicaciones, así como con plataformas utilizadas para fines formativos. Asimismo, entraron a discusión algunos trabajos de investigación recientes relacionados con el empleo de la tecnología con propósitos educativos. Entre las principales conclusiones, se subrayó la importancia de mantener una sensibilidad hacia los adolescentes en su desempeño y adquisición de habilidades tecnológicas, especialmente cuando no están familiarizados con la gestión del conocimiento a través de dichos medios; si bien, han nacido en una era digital o de inteligencia artificial, las destrezas poseídas por muchos de ellos no han alcanzado un nivel de tipo académico.

Palabras clave: Tecnología, aprendizaje en línea, educación, educación a distancia.

\section{Abstract}

Today, a systemic-holistic educational model is continuously being worked on, which is constantly evolving. In this way, the present study had to understand how Learning and Knowledge Technologies (LKT) contributed to the preparation of high school students in the context of distance education throughout confinement for COVID-19. Used a qualitative approach was following the Constructivist Grounded Theory design. The results highlighted that the TAC favored the students experience in different ways; firstly, because the pedagogical strategies had a more significant impact on the teaching process since various situations were promoted, directing high schoolers to play an active role; second, technological resources allowed easy management of learning through different electronicdigital devices; finally, the use of technologies promoted the development of digital skills through constant interaction with applications, as well as with platforms used for formation purposes. Similarly, some research investigations related to the use of technology for educational environments entered the discussion. Among the main conclusions, the importance of maintaining sensitivity towards adolescents in their performance and acquisition of technological skills is underlined, especially when they are not familiar with knowledge management through these means. However, they were born in a digital or artificial intelligence era, the skills possessed by many of them have not reached an academic level.

Keywords: Cognitive competence, investigative competence, textual competence. 


\section{Introducción}

Hoy en día, se trabaja continuamente con un modelo educativo de tipo sistémico-holístico, el cual se encuentra evolucionando constantemente. De este modo, es indispensable comprender lo necesario que es abocarse a asumir dimensiones de una era tecnológica (Latorre et al., 2018). Por tal, las Tecnologías del Aprendizaje y el Conocimiento (TAC), arraigadas a las Tecnologías de la Información y la Comunicación (TIC), se conciben en forma de una oportunidad acuciante para un replanteamiento pedagógico dentro de un marco organizativo de adaptación curricular. A su vez, dan pauta de superar barreras o conflictos sociales, paralelamente mejorando los procesos formativos a través de una interacción más cercana entre los diferentes actores (González, 2020 \& Luque, 2016).

El utilizar tecnología con propósitos educativos es un panorama completamente nuevo para muchos estudiantes. Efectivamente, en otro tipo de situaciones, los adolescentes podrían ser autónomos, audaces, o creativos al momento de desempeñarse expeditamente con los recursos tecnológico-digitales; sin embargo, no es un hecho general para todos los alumnos (Castro, 2005 \& Granado, 2019). Este es un motivo para pensar en una interacción síncrona, en donde los educadores promuevan estrategias como videollamadas con intención de brindar un acompañamiento más personalizado a los discentes, permitiéndoles esclarecer dudas asociadas con actividades escolares, con el propósito de desarrollar competencias genéricas, disciplinares, o en su caso laborales, desde un proceder didáctico-pedagógico, humanista, flexible, o crítico (Amar, 2017 \& Nivela, 2018).

La Tecnología Educativa se constituyó alrededor de los años cincuenta a partir de las aportaciones de Skinner en el conductismo, en respuesta a factores político-socioculturales, teniendo una participación transcendental con su modelo de enseñanza programada. De este modo, son amplias las perspectivas filosóficoepistemológicas que conforman el nexo educativo-tecnológico; sin embargo, hoy en día el uso de TAC debe determinarse según el contexto, necesidades de estudiantes, así como el paradigma bajo el cual se fije una postura; conductista, constructivista, crítico o social, siempre pugnando por el aprendizaje y bienestar de los educandos (Salas, 2002 \& Zabala et al., 2013).

Los avances de tecnologías han ofrecido oportunidades para que los educadores sean creadores de actividades interactivas. También les han permitido tener acceso a un entorno educativo virtual sin restricciones de lugar, tiempo, o bien replantear metodologías de enseñanza desde una perspectiva tecnológica de TIC, lo cual se viene a traducir como TAC (Alvarez et al., 2020; Castro, 2005; Cavus, 2020; \& Nivela, 2018). En tal respecto, existen contextos sociales de pensamiento progresista en los cuales el avance tecnológico es percibido por ser un progreso humanista, donde las tecnologías frecuentemente aclaman igualdad. No obstante, se enfatiza fuertemente en eliminar inequidades digitales. Aunque los recursos tecnológicos del aprendizaje son pauta de un primer paso inclusivo, podrían representar una amenaza a quienes presentan problemas socioeconómicos (Gorski, 2009 \& Granado, 2019).

En este sentido, las TAC comprenden un vasto repertorio de recursos, al incluir dispositivos electrónicos, contenidos digitales, ordenadores, aplicaciones, páginas web y programas de software, utilizados para generar conocimiento de forma innovadora (Amar, 2017 \& Moya, 2013). Un ejemplo particularmente destacado entre el vasto mundo de TIC-TAC, es la aplicación de WhatsApp, contemplada de alta influencia en distintos contextos sociales, donde el Estado mexicano no es una excepción; incluso, podría distinguirse por ser una red social mayormente empleada en entornos familiares, laborales, y educativos (Pérez-Cruz et al., 2020).

A finales de la segunda década del siglo $\mathrm{XXI}$, las TAC pasan a emprender un papel trascendental a raíz del periodo pandémico del COVID-19, el cual forzó al sistema educativo mexicano a entrar en una larga etapa de confinamiento, forzando instituciones a migrar a un esquema de educación remota, en línea, o a distancia, por suscitarse particularmente en un contexto de gran diversidad.

Frente al encierro precautorio para disminuir los contagios del COVID-19, la sociedad se vio emergida en un nuevo estilo de vida con una característica particular, combinándose dentro de un mismo tiempo y 
espacio educación, trabajo, así como responsabilidades del hogar. En el caso de los educadores, existe alta evidencia de un fortalecimiento sobre el uso de recursos tecnológicos aplicados en el ámbito educacional, influyendo altamente por ser un elemento de mediación ante al aislamiento preventivo obligatorio. Desde un punto de vista críticoprogresista, el periodo pandémico permitió elevar ciertas habilidades tecnológicas en miembros de comunidades educativas, abriendo puerta a nuevos espacios de capacitación en instituciones formativas (Sandoval, 2020 \& Mujica-Sequera, 2020).

En tal panorama, los educandos se vieron en urgencia de movilizar habilidades adquiridas a lo largo de su vida con el afán de afrontar nuevas circunstancias que enmarcaron una forma de aprender muy distinta a una modalidad presencial. Planteado el entorno de este trabajo, el objeto de estudio de esta investigación es el empleo de las TAC a modo de recurso predominante en el proceso formativo en un esquema de educación a distancia en tiempos de confinamiento social, de tal manera que se puedan describir las experiencias vividas por los alumnos. Dicho lo anterior, se determinó la siguiente interrogante central como guía de indagación: ¿Cómo contribuyó el uso de TAC en la experiencia educativa de los adolescentes a lo largo del periodo pandémico del COVID-19?

Esta obra se organiza primeramente introduciendo el planteamiento del problema, determinando como objetivo general el comprender el modo en que el uso de los recursos tecnológicos contribuyó en la experiencia de jóvenes cursando el bachillerato en una modalidad emergente a distancia durante el periodo de confinamiento por coronavirus. Después, se presenta el proceso metodológicoindagatorio del objeto de estudio, describiendo resumidamente el procedimiento de examinación de los datos. Posteriormente, se desglosan los hallazgos, explicando las categorías teóricas identificadas. Adicionalmente, en el apartado de discusión se rescatan algunos estudios recientes con el propósito de contrastar resultados. Finalmente, se comparten conclusiones y reflexiones derivadas del abordaje del fenómeno.

\section{Metodología}

La indagación se realizó dentro de los principios emanados tradicionalmente en el enfoque cualitativo, ante lo cual se siguió el diseño de Teoría Fundamentada constructivista (Figura 1). Es un método de investigación preponderante, influenciado por el razonamiento tanto inductivo como abductivo, el pragmatismo, e interaccionismo simbólico, que permite construir teorías fundamentadas sobre un fenómeno estudiado a partir de una combinación de realidades y experiencias de los participantes, las cuales se reflejan en los datos e interpretaciones del investigador, plasmados en sus registros de campo (Brayant, 2017; Charmaz, 2006; Corbin \& Strauss, 2015; \& Glaser \& Strauss, 1967).

\section{Figura 1}

Representación del proceso metodológico de la Teoría Fundamentada

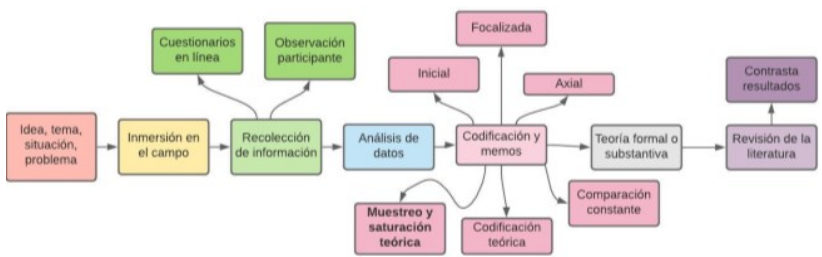

Nota. El diseño de Teoría Fundamentada constructivista es un método altamente flexible, la revisión de literatura o marco teórico entran en segundo término para contrastar resultados, aunque esto no significa que sea menos riguroso, elaboración propia (2020).

Se recolectó información mediante un cuestionario en línea con el objeto de no arriesgar el bienestar de los educandos durante el periodo de confinamiento por COVID-19. De igual modo, se recurrió a la observación participante, así como también a una reflexión constante tomando nota en una bitácora digital editada en un procesador de textos. En este sentido, participaron 23 estudiantes; 18 mujeres y 5 hombres, en un rango de edad entre los 15-18 años, inscritos en el Colegio de Bachilleres del Estado de Sinaloa en Culiacán, México. Según las bases metodológicas, se apeló al muestreo noprobabilístico-intencional, realizándose una selección de manera voluntaria y anónima de discentes, procurando que proporcionaran respuestas enriquecedoras para construir una teoría substantiva (Arias, 2012; Cohen et al., 2018; Creswell, 2012; Leavy, 2017; Naupas et al., 2014; \& Trujillo et al., 2019). 
El análisis de la información se llevó a cabo por medio de un procesador de textos en Microsoft Word. Este procedimiento se considera válido para examinar los registros hechos durante indagaciones de corte cualitativo, o en su caso, de Teoría Fundamentada (San Martín-Cantero, 2014). De tal modo, se realizó una codificación inicial, focalizada, y axial. Adicionalmente, se empleó el método de comparación constante, contrastando códigos, categorías, así como también categorías con códigos; simultáneamente, se elaboró un mapa mental integrando los datos empíricos. Asimismo, se puso en acción el muestreo teórico a fin de fortalecer e iluminar los límites de las categorías, lo cual se tradujo en una saturación teórica. Concurrentemente, se apeló al razonamiento de tipo abductivo con el propósito de interpretar los significados, realidades, y experiencias de los participantes.

\section{Resultados}

El abordaje metodológico del diseño de Teoría Fundamentada constructivista permitió reflejar los siguientes valores teóricos. Las TAC contribuyen positivamente en la experiencia educativa de los educandos de distintas formas. Primeramente, permiten un mejor impacto de las estrategias pedagógicas, dado que se fomentan diversas situaciones de aprendizaje que conllevan al discente a desempeñar un papel activo en su gestión del conocimiento. En segundo lugar, los recursos tecnológicos facilitan administrar el aprendizaje a través de diferentes dispositivos electrónico-digitales, una mayor organización, así como más facilidad de elaboración y edición de tareas. Por último, se promueve el desarrollo de competencias digitales mediante una interacción constante de los estudiantes con aplicaciones y plataformas educativas (Figura 2).

\section{Desarrollo de Competencias con las TAC}

Se detectó que un buen número de discentes tienen capacidad de utilizar las TIC para fines sociales o distintos al área educativa. Afortunadamente, el periodo de confinamiento dentro del marco de una educación a distancia encaminó a los jóvenes a descubrir el papel fundamental de los recursos tecnológicos en su proceso de aprendizaje. De este modo, lo indicó el siguiente alumno:

...he aprendido mucho sobre temas que no sabía. Por ejemplo, saber manejar mejor las aplicaciones de Word y PowerPoint, ya que no sabía usarlas muy bien, y con las clases he aprendido a profundidad. Siempre es muy interesante aprender novedades sobre herramientas digitales (Estudiante 18).

\section{Figura 2}

\section{El papel de las TAC en la educación media} superior

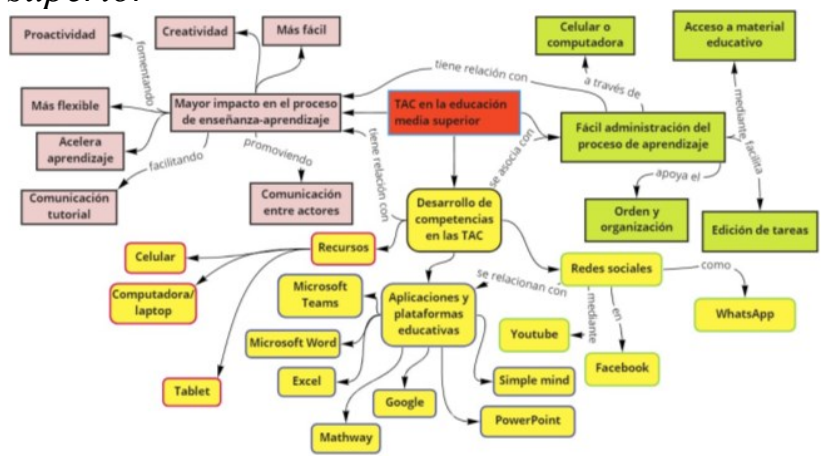

Nota. Las TAC estimulan la activación de distintos aspectos que fortalecen el acto educativo para obtener mayor impacto formativo, desarrollo de competencias tecnológicas, y mayor facilidad de administración del proceso de enseñanza-aprendizaje, elaboración propia (2020).

TAC Utilizadas por Adolescentes. De acuerdo con respuestas obtenidas en el cuestionario en línea, el 100\% de los bachilleres reportaron usar el celular para la realización de tareas. Por otro lado, el ordenador representó una segunda opción, donde el $52.17 \%$ de los educandos manifestó emplear este recurso. Por último, se encontraron las Tablet, un sistema muy similar al teléfono móvil; sin embargo, solo el $0.4 \%$ de los discentes sostuvieron utilizar esta herramienta.

Uso de aplicaciones o plataformas educativas. Según lo expresado por los jóvenes en los cuestionarios en línea, el $91.30 \%$ de los educandos utilizó Microsoft Word para elaboración de sus trabajos escolares. El 43.47\% usó PowerPoint al momento de desarrollar tareas académicas. El 30.43\% empleó diferentes aplicaciones de Google con fines de investigación, así como también de procesamiento de información. El resto de los estudiantes manifestó utilizar recursos de Microsoft Teams, YouTube, WhatsApp, Excel, 
Facebook, OneNote, Writer, y Mathway. En tal sentido, un joven lo argumentó del siguiente modo:

Por ejemplo, Word, OneNote, PowerPoint, u office me ayudan mucho para crear presentaciones de los trabajos que me dejan. Google me ayuda a buscar y encontrar la información que necesito. Adobe Scan me facilita mucho cuando tengo que pasar un apunte de mi libreta a un documento; y, por último, Mathway me auxilia en tareas complicadas de matemáticas (Estudiante 13).

Tabla 1

Tecnologías del Aprendizaje y el Conocimiento empleadas por los bachilleres

\begin{tabular}{cc}
\hline TAC & Porcentaje de estudiantes \\
\hline Uso del celular & $100 \%$ \\
Ordenador & $52.17 \%$ \\
Tablet & $0.4 \%$ \\
Microsoft Word & $91.30 \%$ \\
PowerPoint & $43.47 \%$ \\
Aplicaciones Google & $30.43 \%$ \\
Otros recursos o & $0.3 \%$ \\
plataformas & \\
\hline
\end{tabular}

Nota. Todos los estudiantes cuentan con algún tipo de recurso tecnológico, pero muchos de ellos no cuentan con un grado de competencia suficiente como para emplearlo en su aprendizaje, elaboración propia (2020).

Por otro lado, de acuerdo con el registro de observación participante, se identificó que el recurso, aplicación o plataforma empleado por los alumnos dependió del trabajo académico o competencia a poner en actuación. Igualmente, los adolescentes subrayaron una necesidad de recurrir al uso de distintas aplicaciones o plataformas educativas con el objetivo de llevar a cabo sus actividades de aprendizaje. El estudiante 6 lo expresó así: “...utilizo para investigar Google, mis tareas las hago en libreta o a veces por PowerPoint o por Word".

\section{Mayor Impacto en la Enseñanza y Aprendizaje}

Uno de los aspectos más relevantes descubiertos a través de la observación participante y los datos empíricos de los cuestionarios en línea, es que las TAC contribuyeron de forma contundente en el proceso de aprendizaje, sobre todo aportando al desarrollo de educandos proactivos. Estos detalles se desglosan en las subcategorías presentadas enseguida.
Mayor flexibilidad. Un aspecto crucial detectado fue la flexibilidad facilitada por recursos como el celular, la computadora y Tablet, en el proceso de enseñanza-aprendizaje, al facilitar el acceso, realización, e interacción con actividades educativas. A continuación, se presenta una declaración en torno a tal particularidad: “...pues, en verdad, sí me han ayudado bastante, debido a que gracias a los recursos tecnológicos llevo una mejor educación... más sencilla" (Estudiante 4). Otro alumno sostuvo una respuesta muy parecida: “...el celular me ayuda porque facilita el aprendizaje. Al utilizarlo es un poco más fácil de trabajar. Las tareas que se me facilitan más son las investigaciones porque veo muchísima más información y aprendo más" (Estudiante 11).

Facilidad en comunicación tutorial. Las TAC han traído consigo muchas ventajas, especialmente en el contexto del confinamiento ocasionado por el periodo de pandemia del coronavirus. A manera de ejemplo, se facilitó la comunicación de acción tutorial entre estudiante-estudiante y docente-discente, dando pauta a una atención estudiantil más personalizada, incluso pensada desde un enfoque inclusivo, activando a aquellos bachilleres sobresalientes con el objetivo de apoyar a los adolescentes de bajo rendimiento académico. En seguida, se comparte lo que recalcó uno de los discentes en este respecto:

La tecnología me ha dado experiencia al trabajar ejercicios prácticos, ayuda en las actividades virtuales, progreso en el aprendizaje, me ayuda a corregir cualquier error que tenga con la ayuda más mínima de un docente; también, puedo brindar ayuda $\mathrm{y}$ apoyo mutuo en el cumplimiento de cualquier actividad (Estudiante 15).

Mejor comunicación entre actores. Uno de los aspectos revelados en la observación participante e información analizada en respuestas obtenidas por parte de los discentes, fue una mayor facilidad y sencillez de comunicación promovida mediante los recursos tecnológicos entre los diferentes actores; educando-educando, docente-docente. Es fundamental señalar que la interacción varió según los fines o medios, llevándose a cabo de forma asíncrona o síncrona, con el propósito de 
asignar tareas, acciones tutoriales, o bien, clases síncronas a través de una plataforma virtual. Así lo reportó el estudiante 8: “...la tecnología me ha sido útil para ver las clases, o comunicarme mediante los grupos de WhatsApp cuando hay algún problema. Los cuestionarios en línea también se me han facilitado". Otro joven, expresó un comentario similar:

Como es tiempo de pandemia, la tecnología me ha ayudado a comunicarme con mis maestros. Cuando tengo dudas, consulto algunas páginas en internet para entender mejor o saber más acerca del tema. Me ha facilitado en experimentos de laboratorio, traducir palabras en inglés, y buscar información a utilizar en reportes de literariedad o alguna exposición en cualquier materia (Estudiante 15).

Facilidad en el proceso de aprendizaje. El cuestionario en línea permitió a los jóvenes expresar el modo en que el utilizar recursos tecnológicos ha facilitado su aprendizaje en gran medida. No obstante, se subrayó por muchos de ellos la preferencia sobre el esquema de estudio presencial. Un educando reiteró tal sentimiento como se muestra a continuación: “...el usar aparatos electrónicos básicamente me facilitó elaborar mucho más rápido mis trabajos, pero sigo creyendo que son mejores las clases presenciales" (Estudiante 22). De igual forma, un estudiante agregó un comentario equivalente:

Me ha sido benéfico porque he podido aprender de forma más rápida, porque el teléfono siempre lo tengo en mano. Además, es un dispositivo que cualquier persona puede utilizar de manera sencilla. Adicionalmente, manteniendo una conexión a internet se pueden realizar múltiples actividades como las videollamadas o simplemente investigar algún tema (Estudiante 3).

Fomentando proactividad. Los recursos tecnológicos como el celular permitieron a los educandos tomar más responsabilidad en su proceso de aprendizaje, jugando un papel con mayor independencia en la generación de conocimiento. Un joven lo comentó de la siguiente forma: “...gracias al internet o la web, sin ella no podría entregar mis trabajos, por la razón que está llena de información valiosa. A veces nos dejan temas un poco complejos; entonces, voy y busco información sobre dicho tema" (Estudiante 19). El adolescente a continuación complementó con este planteamiento:

\begin{abstract}
Los recursos tecnológicos como la computadora y celular nos ayudan bastante porque nos facilitan el proceso educativo, aunque no tanto, pero en parte sí, ya que en investigaciones documentales obtenemos información más amplia. Así, al conectarnos en línea, aprendemos mucho más (Estudiante 11).
\end{abstract}

Aprendizaje de forma acelerada. Las TAC permitieron que los procesos educativos se realizaran de forma acelerada. Según los datos empíricos e interpretaciones, esto sucedió especialmente debido a la constante interacción con el teléfono móvil para gestionar información acerca de temas escolares. De este modo, lo planteó el estudiante 12: “...yo soy de lento aprendizaje, pero gracias a recursos digitales como las aplicaciones, aprendo más rápido".

El estudiante 3 agregó un argumento parecido, “...gracias a los recursos tecnológicos pues, se me hacen más sencillos los trabajos que me dejan los maestros, además puedo trabajarlos desde cualquier lugar de una forma más rápida y presentable". La información que proporcionó este discente permitió comprender como los diferentes recursos digitales benefician desde su portabilidad, dando oportunidad de continuar con los estudios desde cualquier ubicación geográfica.

\section{Facilidad de Administración del Proceso de Aprendizaje}

Las TAC brindan un universo de posibilidades para el acceso tanto al aprendizaje como al conocimiento, facilitando una mejor administración del proceso de aprendizaje. Afortunadamente, existen diversas maneras, plataformas, herramientas, y dispositivos, que permiten organizar fácilmente la interacción educativa a distancia. Las subcategorías descritas a continuación vislumbran esta concepción.

Administración mediante dispositivos u ordenadores. El celular, como principal dispositivo administrador de actividades educativas en el presente contexto, sirvió a los jóvenes para descargar distintos tipos de 
aplicaciones, permitiéndoles desarrollar sus tareas. Por otra parte, existieron ocasiones donde los dispositivos de ciertos educandos no tuvieron capacidad de manejar los programas de software o aplicaciones requeridos por los profesores, orillándolos a utilizar el ordenador a modo de alternativa. De esta forma, lo subrayó el siguiente adolescente: “...el teléfono celular es el único recurso tecnológico que me ha ayudado a entregar las actividades y tareas solicitadas por los profesores, tanto a elaborarlas como a enviarlas por aplicaciones como Teams" (Estudiante 14). De igual modo, otro discente agregó un comentario parecido:

\begin{abstract}
...el celular se me ha hecho la herramienta más rápida y básica, ya que a dónde vaya lo llevo conmigo y puedo llevar a cabo investigaciones o varias actividades que me piden. Además, puedo mandarlas o descargar las aplicaciones necesarias. De igual forma, utilizo la computadora en caso de que algunas aplicaciones no tengan más funciones, para que no me falte ningún trabajo (Estudiante 9).
\end{abstract}

Acceso a material educativo. A pesar de que la educación está sumergida en un contexto de diversidad, las TAC jugaron un papel sustancial para el aprendizaje de los alumnos al permitirles el acceso a contenido digital desarrollado por sus profesores, o bien, a otras fuentes proporcionadas a través de los distintos medios de comunicación. En este sentido se reportó:

Los libros digitales me ayudaron mucho al inicio del semestre, ya que no contaba con los libros de la preparatoria. Los videos me ayudan, dado que, si no comprendo un tema, busco videos de explicaciones, también las planillas digitales, puesto que así los maestros hacen los exámenes para responderlos (Estudiante 13).

Facilidad de edición de trabajos. Las TAC ofrecieron distintas formas de editar las tareas desarrolladas por los estudiantes, permitiendo una corrección y procesamiento más sencillo del conocimiento. Muchos de los trabajos de aprendizaje que realizaron los discentes se produjeron de manera artesanal, en otras palabras, a mano, contestando algún libro impreso o bien, dando cuenta de alguna evidencia elaborada como manualidad, dependiendo de la disciplina o competencia a desarrollar. Así se destacó en el comentario a continuación:

\begin{abstract}
...estas aplicaciones son de gran utilidad, pues facilitan la realización de trabajos, además de que son fáciles de utilizar. Lo que viene siendo Microsoft Word, es una herramienta muy útil y sencilla de usar, por el motivo de que no es laboriosa. Me ha servido mucho para dar formato a mis actividades, me he quitado de estar escribiendo hojas y hojas, es más sencillo colocar en un archivo todo (Estudiante 9).
\end{abstract}

Orden y organización. El uso de las TAC permitió a los discentes llevar un orden en su proceso de aprendizaje, porque los diferentes recursos tecnológico-digitales fomentaron una mayor organización con sus trabajos. De esta manera, lo comentó el siguiente discente: “...los recursos digitales me han sido benéficos. Al momento de utilizarlos, puedo elaborar mis trabajos de forma más ordenada y clara, ya que no se me enreda la información y comprendo todo mejor" (Estudiante 2). Existió otro alumno que también se expresó de forma parecida:

\begin{abstract}
$\mathrm{Me}$ ha beneficiado en organizar mi información, puesto que tengo separadas las tareas por nombres, y a la hora de entregar lo que me hayan dejado pues solo selecciono el archivo con el nombre que le puse para mandarlo a la materia correspondiente (Estudiante 10).
\end{abstract}

\section{Otros Resultados}

A pesar de las numerosas ventajas $\mathrm{y}$ aspectos positivos que ofrecen las TAC, algunos estudiantes siguieron prefiriendo el estilo de aprendizaje artesanal, es decir, utilizando material impreso, lápices, plumas, entre otros. El motivo es que los bachilleres se ven desfavorecidos cuando la tecnología falla o presenta problemas técnicos. Adicionalmente, ciertos educandos manifestaron una falta de preparación para utilizar los recursos digitales educativos requeridos por el sistema escolar.

\footnotetext{
...a mí se me hace mucho mejor estar estudiando en la prepa (presencial) que por línea. Sí aprendemos, pero no es la misma,
} 
porque a veces puede que no funcionen nuestros teléfonos, o también, a veces no le entiendo a las aplicaciones o a algunas tareas (Estudiante 6).

\section{Discusión}

Son diversos los estudios relacionados con las Tecnologías del Aprendizaje y el Conocimiento. En este tenor, el estado del arte da cabida para entablar amplia discusión en torno a la aplicación de los recursos tecnológicos en el ámbito educativo. En seguida, se resumen algunas obras de indagación, con el fin de compartir tanto contrastes como reflexiones a partir de los hallazgos derivados de esta investigación, un proceso fundamental dentro del marco metodológico de la teoría fundamentada.

González (2020) emprendió una investigación en el contexto cultural colombiano, cuyo objetivo fue establecer alternativas con el propósito que las TAC contribuyeran a mejorar las condiciones de vida, proyectos educativos, y productivos de los exintegrantes de las Fuerzas Armadas Revolucionarias-Ejército del pueblo (FARC-EP) en su reincorporación social. Se optó por un enfoque cualitativo de corte exploratorio secuencial (Creswell, 2012), realizando un análisis documental, con una encuesta de opinión aplicada a 20 reincorporados. El resultado culminó en una propuesta cuyo objetivo buscó incorporar los recursos tecnológico-digitales dentro del programa formativo de los participantes, indicando aquellos requerimientos académico-operativos necesarios para desarrollar competencias tecnológicas.

En cuanto a los resultados, se puede apreciar como los individuos indagados comparten cierta preferencia por llevar a cabo un proceso educativo de interacción con audiovideo, al igual que los bachilleres del presente. En el mismo orden de ideas, los encuestados respondieron utilizar YouTube, Facebook, o Google al momento de entablar acciones vinculadas con su educación. Igualmente, algunas de estas personas manifestaron habilidades asociadas con el uso de tecnología; sin embargo, al responder en pregunta abierta, jamás expresaron ponerlas en acción con intenciones de beneficiarse educativamente.

De igual modo, desde perspectivas científicas de autores como Verdecia et al.
(2015), la aparición de los diferentes medios, entre ellos el internet, han provocado el surgimiento de distintas formas de acceso al conocimiento. En este respecto, diversas situaciones adversas han forzado al hombre a agotar todos los recursos disponibles en su poder; tecnología, dispositivos digitales, o conectividad a internet, a fin de continuar con el avance social, indispensable para evitar el estancamiento en progresos industriales, científicos, tecnológicos, empresariales, o laborales. El ejemplo perfecto es el periodo de confinamiento originado en el 2019, el cual se prolongó de manera indefinida golpeando la estabilidad de muchas personas, así como también de organizaciones públicas y privadas.

Las TAC han revivido distintos planteamientos teóricos, tal vez el más notable podría ser el aspecto epistemológico sociocultural. Diversos filósofos han reconocido tanto al ser humano como al conocimiento por ser procesos históricos, culturales, sociales, de interacción con otros, como lo expuso en su momento Vygotsky (Vila, 2001), o María Montessori en su visión de educación humanista, libre, y naturalista (Aguilar \& Manzano, 2018 \& Caló, 1956). De esta manera, se interpreta la posibilidad de los bachilleres de desarrollar competencias a partir del contexto cultural que viven, con ayuda de sus pares o maestros, aunque también de aquellos recursos a su disposición. Algunas TAC que son posibles implementar con los discentes en ambientes de gran diversidad o desigualdad desde un enfoque inclusivoinnovador son los blogs, wikis, Google, Ted, YouTube, redes sociales, entre otros (Casablancas, 2014; Cruz, 2014; \& Vera \& Sobenis, 2017).

\section{Conclusiones}

Se considera que se logró una aproximación muy acertada al objeto de estudio de esta indagación, lo cual fue posible gracias a las orientaciones metodológicas entabladas principalmente dentro del diseño de Teoría Fundamentada constructivista. Por tal, se consiguió alcanzar el objetivo general y comprender como el emplear recursos tecnológicos contribuyó en la experiencia educativa de bachilleres en una modalidad 
emergente a distancia durante el periodo pandémico del COVID-19.

Ahora bien, se pone a juicio de cada institución, centro escolar, o docentes, el continuar con investigaciones que pongan a prueba, en sus contextos particulares, los valores teóricos del presente. En este respecto, pudieran ser diversos los planteamientos o interrogantes guías para futuras indagaciones, en lo particular surgen como preguntas de seguimiento, ¿De qué modo influyen las TAC en el desarrollo de competencias genéricas, disciplinares y profesionales en tiempos del coronavirus?; ¿Cuáles son los efectos de TIC o TAC en el proceder pedagógico de maestros en el periodo de confinamiento?; o, ¿Qué impacto causa el uso de TAC en indicadores académicos?

Las preguntas e hipótesis interpretadas en los hallazgos del presente tienen intención de servir a manera de inspiración para continuar investigando dentro del mismo eje temático, pero pudieran por supuesto emerger otras. Asimismo, es vital recalcar que el abordaje metodológico da cabida a una amplia variedad de indagaciones; el diseño de Teoría Fundamentada es flexible al momento de realizar estudios de carácter deductivo e inductivo, como se puede corroborar en la basta literatura relacionada con sus modelos empírico, sistemático, o constructivista.

\section{Reconocimiento}

Un reconocimiento a la Universidad Autónoma de Occidente y al Instituto de Estudios Superiores en Educación por Competencias al facilitar los fundamentos para realizar investigación en educación.

\section{Referencias}

Aguilar, N. L. \& Manzano, N. (2018). La mentoría en el nivel universitario: Etapas para su implementación. Universidad y Sociedad, 10(1), 255-262. http://rus.ucf.edu.cu/index.php/rus

Alvarez, A. V., Ventura, D. R. M., \& Opiniano, J. O. Going the distance: perceptions of teachers in open and distance continuing professional development. Globus Journal of Progressive Education, 10(20), 60-66. https://www.globusedujournal.in/wpcontent/uploads/2020/11/GE-JD207-Abel-V.-Alvarez.pdf

Amar, V. M. (2017). La importancia de la TAC en la educación y cambios sociales. Revista De Estudios Socioeducativos. ReSed,

(5),

$16-28$
https://revistas.uca.es/index.php/ReSed/article/view/S.1.1 N5

Arias, F. G. (2012). El Proyecto de Investigación: Introducción a la metodología científica (sexta edición). Editorial Episteme, C.A

Brayant, A. (2017). Grounded theory and grounded theorizing: Pragmatism in research process. Oxford University Press.

Caló, G. (1956). Maria Montessori. En Château, J. (dir), Moreau, J., García, V., Mesnard, P., Piobetta., Mallinson, V., J. B., Meylan, L., Flitner, W., Savioz, R., Plancke, R., Dottrens, R., \& Brubacher, J. S. Los grandes pedagogos. Fondo de Cultura Económica.

Casablancas, S. (2014). De las TIC a las TAC, un cambio significativo en el proceso educativo con tecnologías. Virtualidad, educación y ciencia, 5(9), 106-109. https://revistas.unc.edu.ar/index.php/vesc/article/view/99 26

Castro, P. (2005). Estrategia de integración de la alfabetización electrónica a la enseñanza-aprendizaje del inglés con fines especificos en la carrera de ingeniería agronómica [Tesis de doctorado, Universidad Central Martha Abreu De Las Villas].

https://www.researchgate.net/publication/276983422_Es trategia_de_integracion_de_la_alfabetizacion_electronic a_a_la_ensenanzaaprendizaje del ingles con fines especificos en la ca rrera_de_ingenieria_agronomica/link $/ 555 \mathrm{cb} 68 \overline{\mathrm{d}} 08 \overline{\mathrm{a}} \overline{\mathrm{i}} 8 \mathrm{~B} \mathrm{c}$ $06 \mathrm{~b} 5 \mathrm{~d} 3 \mathrm{e} 80 /$ download

Cavus, N. (2020). Evaluation of MoblrN m-learning system: Participants' attitudes and opinions. World Journal on Educational Technology: Current Issues, 12(3), 150164. https://doi.org/10.18844/wjet.v12i3.4978

Charmaz, K. (2006). Constructing grounded theory: A practical guide through qualitative analysis. Sage Publications.

Cohen, L., Manion, L., \&Morrison, K. (2018). Research Methods in Education (octava edición). Routledge.

Corbin, J. \& Strauss, A. (2015). Basics of qualitative research: Techniques and procedures for developing grounded theory (fourth edition). Sage Publications.

Creswell, J. W. (2012). Educational research: Planning, conducting, and evaluating quantitative and qualitative Research (fourth edition). Pearson Education, Inc.

Cruz, V. (2014). Nivel de competencias y actitudes hacia las TIC por parte de los docentes de los centros educativos en República Dominicana. Acercamiento a dos casos [Tesis de Maestría, Universidad de Salamanca].

https://gredos.usal.es/bitstream/handle/10366/123431/TF M_MasterTIC_Viane_M_Cruz_Campusano.pdf?sequen $\mathrm{ce}=1$ \&isAllowed $=\mathrm{y}$

Glaser, B. G. \& Strauss, A. 1. (1967). The Discovery of grounded theory: strategies for qualitative research. Aldine.

González, M. P. (2020). Tecnologías del Aprendizaje y el Conocimiento para el Trabajo y Desarrollo Humano de los reincorporados del Espacio Territorial de Capacitación y Reincorporación de Tierra Grata, Cesar 
[Tesis de Maestría, Universidad Militar Nueva Granada]. Repositorio de la UMNG: http:/hdl.handle.net/10654/36136.

Gorski, P. C. (2009). Insisting on Digital Equity: Reframing the Dominant Discourse on Multicultural Education and Technology. Urban Education, 44(3), 348-364. https://doi.org/10.1177/0042085908318712

Granado, M. (2019). Educación y exclusión digital: los falsos nativos digitales. Revista de Estudios Socioeducativos. ReSed, (7), 27-41.

https://revistas.uca.es/index.php/ReSed/article/view/440 4

Latorre, E. L., Castro, K. P., \& Potes, I. D. (2018). Las TIC, las TAC y las TEP: Innovación educativa en la era conceptual. Universidad Sergio Arboleda. Repositorio de la USA https://repository.usergioarboleda.edu.co/handle/1123 $2 / 1219$

Leavy, P. (2017). Research design: Quantitative, qualitative, mixed methods, arts-based, and community-based participatory research approaches. The Guildford Press.

Luque, F. (2016). Las TIC en educación: caminando hacia las TAC. 3C TIC. Cuadernos De Desarrollo Aplicados a Las TIC, 5(4), 55-62.

https://doi.org/10.17993/3ctic.2016.54.55-62

Moya, M. (2013). De Las TICs a Las TACs. La Importancia De Crear Contenidos Educativos Digitales. DIM: Didáctica, Innovación Y Multimedia, (27), 1-15. http://dim.pangea.org/revistaDIM27/docs/AR27conten idosdigitalesmonicamoya.pdf

Mujica-Sequera, R. M. (2020). La Enseñanza Tecnoemocional en la Educación del Siglo XXI. Revista TecnológicaEducativa Docentes 2.0, 9(2), 71-78. https://doi.org/10.37843/rted.v9i2.147

Nivela, A. (2018). Tecnologías del Aprendizaje y Conocimiento (TAC) en el aprendizaje significativo de los estudiantes del octavo año de educación general básica jornada matutina de la unidad educativa "Provincia de Cotopaxi”. Diseño de software educativo. [Trabajo de pregrado, Universidad de Guayaquil]. Repositorio UG: http://repositorio.ug.edu.ec/handle/redug/36947

Ñaupas, H., Mejía, E., Novoa, E., \& Villagómez, A. (2014). Metodología de la investigación cuantitativa-cualitativa y redacción de la tesis (cuarta edición). Ediciones de la $\mathrm{U}$.

Pérez-Cruz, D., Sánchez-López, F., Cocón-Juárez, J. F., \& Zavaleta-Carrillo, P. (2020). La Influencia del WhatsApp en la Educación Superior de la UNACAR. Revista Tecnológica-Educativa Docentes 2.0, 9(2), 3948.

https://doi.org/10.37843/rted.v9i2.143

Salas, F. E. (2002). Epistemología, educación y tecnología educativa. Revista Educación, 26(1), 9-18. https://www.redalyc.org/pdf/440/44026102.pdf

Sandoval, C. H. (2020). La Educación en Tiempo del Covid-19 Herramientas TIC: El Nuevo Rol Docente en el
Fortalecimiento del Proceso Enseñanza Aprendizaje de las Prácticas Educativa Innovadoras. Revista Tecnológica-Educativa Docentes 2.0, 9(2), 24-31. https://doi.org/10.37843/rted.v9i2.138

San Martín-Cantero, D. (2014). Teoría fundamentada y Atlas.ti: recursos metodológicos para la investigación educativa. Revista electrónica de investigación educativa, 16(1), 104-122.

http://www.scielo.org.mx/scielo.php?script=sci_arttext\& pid $=$ S1607-40412014000100008\&lng=es\&tlng=es

Trujillo, C. A., Naranjo, M. E., Lomas, K. R., \& Merlo, M. R. (2019). Investigación Cualitativa: Epistemología, Consentimiento Informado, Entrevistas en Profundidad (1ra ed.). Universidad Técnica del Norte.

Vera, G. M. \& Sobenis, D. E. (2017). Las tecnologías de aprendizaje y Conocimientos (TACs) en el Rendimiento Académico. [Trabajo de pregrado, Universidad de Guayaquil]. Repositorio de la UG: http://repositorio.ug.edu.ec/handle/redug/25545

Verdecia, E., Enríquez, S. C., Gargiulo, S. B., Ponz, M. J., Scorians, E. E., Vernet, M. \& Wenk, N. (2015). Information and comunications technologies in education. Curren achievements and future prospects. Revista Cubana de Educación Superior, 34(2), 4-17. http://scielo.sld.cu/scielo.php?script=sci_arttext\&pid=S0 257-43142015000200001\&lng=es\&tlng=en.

Vila, I. (2001). Lev S. Vigotsky: La psicología cultural y la construcción de la persona desde la educación. En Trilla, J. (coord.), Cano, E., Carretero, M., Escofet, A., Fairstein, G., Fernández, J. A., González, J., Gros, B., Imbernón, F., Lorenzo, N., Monés, J., Muset, M., Pla, M., Puig, J. M., Rodríguez, J. L., Solà, P. Tort, A., \& Vila, I. El legado pedagógico del siglo XX para la escuela del siglo XXI (pp.207-227). Editorial Graó, de IRIF, S.L.

Zabala, C., Camacho, H., \& Chávez, S. (2013). Tendencias epistemológicas predominantes en el aprendizaje de las TIC en el área de la educación. TELOS. Revista de Estudios Interdisciplinarios en Ciencias Sociales, 15(2), 178-194. https://biblat.unam.mx/es/revista/telos-revistade-estudios-interdisciplinarios-en-cienciassociales/articulo/tendencias-epistemologicaspredominantes-en-el-aprendizaje-de-las-tic-en-el-areade-la-educacion 